\title{
LETTERS
}

doi:10.1017/S1041610206214029

\section{Boundary crossing and boundary violation by service providers and carers in dementia care}

The metaphor or paradigm of a boundary separating a professional's role, identity, needs, wishes, desires and actions from those of their patient or client has gained increasing currency in recent years (Gabbard and Nadelson, 1995). Boundary crossings and violations by professionals can be associated with or lead to sexual, financial and verbal abuse, domination and control of clients or patients.

To our knowledge, issues associated with boundary crossing and violation as they relate to dementia care have not previously been raised in refereed literature. Nor have they been raised in relation to service providers' and carers' working relationships with people with dementia, where these service providers and carers are not deemed to be "professionals". Peterson (1992), in a landmark book, applied the concept of boundaries to relationships between professionals and clients generally. Gutheil and Gabbard (1993) described and explored boundary theory in relation to psychotherapy and psychiatry. Gabbard and Nadelson (1995) extended the discussion to physician-patient relationships. We would like to draw more attention to the boundary concept and the associated useful body of boundary theory in order that these be more widely promoted in education, training, supervision and the development of protocols in a wide range of relationships of trust between carers and workers involved in dementia care, or involved in providing services to those experiencing dementia and other cognitive impairment. We feel that the concept can be usefully applied to a wide and disparate range of persons, some of whom might not be considered as "professionals" but who are in some form of helping or caring role. These people include: carers both paid and unpaid, volunteers, family members and friends, those providing home care and home maintenance services, registered and enrolled nurses, assistants in nursing, personal care assistants, pastoral care workers, social workers, psychologists, diversional therapists, occupational therapists, physiotherapists, clergy, medical practitioners (including psychiatrists), lawyers and accountants.

Interactions between a professional and a client are inevitably associated with a power asymmetry or power differential (Peterson, 1992). In working with a cognitively-impaired client, many people who may not usually be defined as professionals (e.g. tradesmen, neighbours) are placed in a relationship with the 
same potential or actual power asymmetry, with associated risks of boundary violation.

We would like to see assessment of and education about boundary issues incorporated into the process of assessment and management of individuals who are cognitively impaired and in concurrent education and support for their carers. The concept can be particularly useful in recognizing and understanding elder abuse and neglect. For instance, ignorance about boundaries amongst wellmeaning care workers can place them at higher risk of burnout or exploitation by clients or by the care workers' employers, and even lead to reciprocal elder abuse.

Boundary issues can also be very significant when considering substitute decision-making associated with late-life and end-of-life decisions, where boundary crossing is unavoidable. Several writers (Gutheil and Gabbard, 1993, Gabbard and Nadelson, 1995, Gutheil and Gabbard, 1998) have distinguished between boundary crossings and boundary violations. In boundary violations, a professional abuses the privilege of a relationship of trust, often to serve their own needs rather than the needs of their client. In contrast a boundary crossing in psychotherapy may be "a benign variant, where the ultimate effect of the deviation (from usual boundary rules) may be to advance the therapy in a constructive way that does not harm the patient". These authors also describe as boundary crossings: entirely appropriate human responses to unusual events, such as a therapist's response to a patient falling, or a physician holding the hand of a patient who has just lost a family member and who reaches out to the physician. But they urge an awareness by the therapist and physician of the possible boundary issues even in such apparently simple situations, the danger that even apparently benign boundary crossings can damage or derail a therapeutic alliance, can lead down the "slippery slope" to boundary violations and can be used in legal proceedings as evidence of an inappropriate relationship between a therapist or physician and a client or patient. There are also professionals and others who are predatory in their behavior and who use boundary crossings as a prelude to boundary violations, a process which has been described as "grooming" (Brown, 2003). Most authors urge that the concept of boundaries should not simply and only lead to rigid rules or guidelines, but that the significance of particular boundary crossings can only be assessed by careful attention to the clinical context. We consider that the same broad principles apply to boundary crossing and boundary violation in service provider- client relationships. In dementia care, boundary crossings by either service provider or client are not necessarily problematic but generally need to be recognized and reviewed. The process of reviewing such incidents is in itself a powerful tool to improve care and to reduce risks.

The literature on boundaries speaks not only of boundary crossings by professionals, but of boundary crossings by clients - and this occurs in dementia 
care also. Some cognitively and behaviourally-impaired clients cross boundaries easily themselves and have a higher than usual potential to invite, allow or even demand boundary crossing by a service provider. Recognition of this potential can reduce difficulties for the service provider.

As in psychotherapy, psychiatry and medicine, the concept of boundaries can be useful, not to provide an arbitrary set of rules, or to dehumanize helpful relationships between service providers and people with cognitive difficulties, but rather as a metaphor which can be kept prominently in mind to avoid some of the pitfalls and reduce some of the challenges of caring, helping and interacting with cognitively-impaired individuals. Boundary theory can lead to protocols, rules and guidelines for service providers but these should be accompanied by education, training, supervision, mentoring, and increased self-awareness among service providers, if only because not every nuance of every real life situation can be covered by a set of rules.

\section{References}

Brown, H. (2003). The role of the Public Guardianship Office in safeguarding vulnerable adults against financial abuse. What is financial abuse? (Report) London: Public Guardianship Office. www.guardianship.gov.uk/downloads/FinancialAbuseResearchreportsummary.doc

Gabbard, G. O. and Nadelson, C. (1995). Professional boundaries in the physician-patient relationship. $\mathcal{F} A M A, 273,1445-1449$.

Gutheil, T. G. and Gabbard, G. O. (1993). The concept of boundaries in clinical practice: theoretical and risk-management dimensions, The American fournal of Psychiatry, 150, $188-196$.

Gutheil, T. G. and Gabbard, G. O. (1998). Misuses and misunderstandings of boundary theory in clinical and regulatory settings, The American fournal of Psychiatry, 155, 409-414.

Peterson, M. (1992). At Personal Risk: Boundary Violations in Professional-Client Relationships. New York: W. W. Norton \& Company.

Sid Williams, ${ }^{1}$ Anne Sammut ${ }^{2}$ And Wendy Blaxland ${ }^{3}$

${ }^{1}$ PO Box S 35, Homebush South, NSW 2140, Australia

Email: gertboyzee@bigpond.com.au

${ }^{2}$ Alzheimer's Australia NSW, Australia

${ }^{3}$ Domiciliary Care, Geriatric Medicine, Westmead Hospital, NSW, Australia

\section{Getting lost for the first time in patients with Alzheimer's disease}

Getting lost is an incipient symptom for some patients with Alzheimer's disease (AD) (Pai et al., 2004), which becomes more frequent as the disease progresses. Such patients often exhibit hesitation or confusion and almost always lose the 
autonomy to navigate freely. As the disease progresses, they become unable to find their way home without help from others, and finally will be confined, or will confine themselves, in places in which their wandering may be safely managed. This problem worries not only the patient, but also casts a heavy burden on the family. We conducted a study with the aim of describing the context in which patients first became lost.

The study was carried out in a dementia clinic in southern Taiwan. We defined "getting lost" (GL) as a condition in which a patient found him/herself unable to find the way to a destination or to return home or to where he/she started from. Escorted home by others (EHBO) is one of the consequences to GL when getting home is impossible without help. Not all GL patients need EHBO, if they can make a phone call, hire a cab or use other methods to find their way home. We administered a semi-structured questionnaire to the patient and family, focusing on the context in which the patient's first GL had occurred, including the place, destination or purpose of the journey and the means of transportation.

Nineteen participants completed the study, 10 male and 9 female, with a mean age of $70(\mathrm{SD}=8)$ years and formal education of $6(\mathrm{SD}=5)$ years. The duration of illness was $58(\mathrm{SD}=40)$ months. Their Cognitive Ability Screening Instrument scores averaged $56(\mathrm{SD}=23)$ and Mini-mental State Examination score (MMSE) $16(\mathrm{SD}=7)$. No significant changes in the patient's environment over the past 5 years were found in 17 patients. The last move occurred within 10 years for six patients, 10 to 20 years ago for seven, while six of them had never moved. The table $S 1$, published as supplementary material online attached to the electronic version of this letter at www.journals.cambridge/jid_IPG, shows the context of the first GL events. Action taken by the family or caregivers in response to the first GL included informing neighbors (8), having the patient wear a wrist pendant or similar (7), putting him/her in a nursing home (3), giving the patient a map (1), and doing nothing (6).

In this study, most of the patients had their first GL within two years after the onset of AD. This result suggests a central role for the hippocampus in human navigation (O'Keefe et al., 1978; Maguire et al., 1999), which is damaged early in the clinical course of AD. Seventeen of the 19 patients $(89 \%)$ first became lost in a place they had been familiar with for more than a decade. This finding is inconsistent with our expectation and with those described in previous reports (Cherrier et al., 2001; Maguire et al., 1999). Most caregivers reported that, upon feeling lost or disoriented, the early AD patient under their care would attempt to retrace the route home. The sense of becoming lost may confine patients to increasingly restricted territorial ranges, or "niches" during the early part of the disease. As the disease progresses, and as the explicit sense of being lost either expands to most of the territorial range, or the sense disappears altogether, this confinement may gradually disappear. As a result, $\mathrm{AD}$ patients may enter 
territory that would ordinarily be unfamiliar to them, and become truly lost. These patients may no longer be aware of a difference between "familiar" and "unfamiliar" environmental contexts, because differentiating such contexts, no matter how trivial or drastic, depends on intact hippocampal functions. The pathological lesions in the hippocampus and related structures in early AD may prevent patients from "updating" current contexts. Even the small inevitable differences between a current environmental context and the same context as stored in memory may confuse patients. A second, major finding is that $89 \%$ of the patients had a definite destination in mind when they first became lost: eight were attempting to return to their present home, and another four were attempting to return to a former home. This goal-directed behavior is in contrast to the scenarios one might expect, such as aimless wandering. Thus, the GL described here is different from that of an adverse effect of medication or nonmedication-related wandering. Many patients reported that the episodes of GL occurred suddenly, for example, after making a turn. They described recognizing landmarks but being unable to find their way efficiently. Instead, they wandered while remaining near the destination. It seemed that the patients could recognize an isolated landmark in familiar surroundings, but their perception of the scene as a whole no longer provoked a feeling of familiarity. That some of them could describe the layouts of the place where they had been lost suggests that they had a fair cognitive map. Such cases also suggest that an intact cognitive map does not guarantee successful navigation. There might be a mismatch, however, between the fair cognitive map and the real-life context. Another possibility is that these patients in fact had applied the "well-worn route" strategy or habitual route (Hartley et al., 2003), which is believed to be dependent on caudate nuclei, which are relatively intact until later stages of $\mathrm{AD}$.

Furthermore, five patients reported that returning home was more difficult than reaching an outbound goal. These patients reported reaching their destination as expected, but became lost on the way back home. It is possible that the two directions, "toward-home" and "away-from-home", involve different mechanisms during human navigation. Finally, the precautions a family took in response to the GL-event varied. Repeated GL-events suggest lack of awareness of the potential risks for the $\mathrm{AD}$ patient or their family. We encourage patients to wear an alert medal even in the early stage of $\mathrm{AD}$, for GL-events may occur unexpectedly.

In summary, the present results represent the situation of patients living in southern Taiwan. Generalization of these results to all early AD patients should be done with some caution. Nevertheless, these findings suggest that, for early $\mathrm{AD}$ patients, familiar places are not as safe as most caregivers may believe. The elucidation of GL-behavior in this study provides important information for patients, their families, and physicians. 


\section{Acknowledgements}

The authors thank Professor W. Jake Jacobs of the Department of Psychology, the University of Arizona, Tucson, for his helpful comments and encouragement.

\section{References}

Cherrier, M. M. et al. (2001). Route-learning performance in Alzheimer's disease patients. Neuropsychiatry, Neuropsychology and Behavioural Neurology, 14, 159-168.

Hartley, T. et al. (2003). The well-worn route and the path less traveled: distinct neural bases of route following and wayfinding in humans. Neuron, 37, 877-888.

Maguire, E. A. et al. (1999). Human spatial navigation: cognitive maps, sexual dimorphism, and neural substrates. Current Opinion in Neurobiology, 9, 171-177.

O'Keefe, J. et al. (1978). The Hippocampus as a Cognitive Map. Oxford: Oxford University Press. Pai, M. C. et al. (2004). Topographical disorientation in community-residing patients with Alzheimer's disease. International fournal of Geriatric Psychiatry, 19, 250-255.

\section{Min-Chien TU ${ }^{1}$ AND MING-Chyi PAI ${ }^{2}$}

${ }^{1}$ Department of Medicine

${ }^{2}$ Division of Behavioral Neurology, Department of Neurology, National Cheng Kung University Medical College, Tainan 704, Taiwan

E-mail: pair@mail.ncku.edu.tw 